\title{
Evaluating the Binding of Selected Biomolecules to Cranberry Derived Proanthocyanidins using the Quartz Crystal Microbalance
}

\author{
Nicole E. Weckman, Adam L.J. Olsson, and Nathalie Tufenkji* \\ Department of Chemical Engineering, McGill University, Montreal, Quebec H3A 2B2, Canada
}

${ }^{*}$ Corresponding author:

Phone: (514) 398-2999; Fax (514) 398-6678

Email: nathalie.tufenkji@mcgill.ca 


\begin{abstract}
Despite cranberry being associated with the prevention of bacterial infections for over a century, our understanding of the bioavailability and mechanisms by which cranberry prevents infection is limited. This study investigates the interactions between cranberry proanthocyanidins (CPAC) and human serum proteins (albumin, $\alpha$-1-acid glycoprotein, and fibrinogen) that may be encountered during CPAC metabolism following ingestion. To better understand how CPAC might interfere with bacterial infection, we also examined the interactions between CPAC and selected bacterial virulence factors; namely, lipopolysaccharide (LPS) and rhamnolipid. The binding of CPAC to the serum proteins, rhamnolipids, and LPS from Escherichia coli O111:B4 can be described by Langmuir-type isotherms allowing the determination of the apparent adsorption affinity constants, with CPAC interacting most strongly with fibrinogen with a binding constant of $2.2 \times 10^{8} \mathrm{M}^{-1}$. These binding interactions will limit the bioavailability of the CPAC at the site of action, an important consideration in designing further clinical trials. Furthermore, CPAC interacts with Pseudomonas aeruginosa 10 LPS, E. coli O111:B4 LPS, and P. aeruginosa rhamnolipids in fundamentally different manners, supporting the theory that cranberry prevents bacterial infections via multiple mechanisms.
\end{abstract}

Keywords: QCM-D, cranberry proanthocyanidins, lipopolysaccharide, rhamnolipid, protein 


\section{INTRODUCTION}

Recurrent urinary tract infections (UTIs) are traditionally prevented using a low dose antibiotic regime; however, this type of antibiotic treatment is strongly associated with the development of antibiotic resistant bacteria ${ }^{1}$. Cranberry, which has been associated for almost a century with the prevention of UTIs ${ }^{2}$, has thus been increasingly researched as a natural and widely available alternative to traditional antibiotic treatment regimes for the prevention of such infections. Despite numerous clinical trials (several of which are inconclusive), our understanding of the bioavailability and the mechanisms by which cranberry may prevent bacterial infection is limited, perhaps due to a lack of understanding of the complex factors affecting the bioavailability and metabolic fate of cranberry derived materials from ingestion to excretion ${ }^{3,4}$.

The bioavailability of the cranberry proanthocyanidin (CPAC) fraction is of particular interest because of its proven ability to modulate the behavior of bacteria 5 . A key factor that can limit the bioavailability of a drug at the site of action is drug binding to plasma proteins such as human serum albumin (HSA), fibrinogen, and $\alpha$-1-acid glycoprotein (AAG). Only unbound drug is free to reach the active site ${ }^{6}$; hence, the binding of cranberry derivatives such as CPAC to serum proteins may limit their bioavailability in the gut, bladder, urinary tract, or other sites of infection. While few studies have been conducted on the binding of proanthocyanidins to model proteins such as bovine serum albumin and selected proteins found in the intestine ${ }^{3,7,8}$, it is important to investigate the binding of CPACs to human serum proteins that could functionally affect CPAC bioavailability following ingestion and metabolism. 
In addition to uncertainty in the bioavailability of CPAC, the mechanism by which it may prevent bacterial infections is not well understood. Understanding the molecular mechanism(s) via which CPAC interacts with various bacteria is crucial for development of technologies taking advantage of those properties; for example, biomedical devices treated with CPAC to prevent device associated infections ${ }^{9,10}$. One mechanism by which cranberry may prevent bacterial infections is by impairing bacterial adhesion to host cells ${ }^{11}$ by altering the bacterial surface properties ${ }^{12}$, morphology and shape ${ }^{13}$, or by interfering with P-type fimbriae of Escherichia coli ${ }^{14,15}$. Another possible mechanism via which CPAC interferes with bacterial virulence is through decreasing the motility (i.e., swimming and swarming) of the bacteria ${ }^{10,16-19}$. It has also been shown that CPAC acts as an iron chelator and limits iron availability to uropathogenic E. coli ${ }^{20}$.

As the primary component of the outer cell membrane of pathogenic Gram-negative bacteria, bacterial lipopolysaccharides (LPS) are required for swarming motility ${ }^{21}$, and are important to both the structure and the overall surface charge of the bacteria ${ }^{22}$. These factors contribute to the importance of LPS as a virulence factor and a cellular adhesion molecule ${ }^{23}$. As such, the binding of CPAC to LPS could play a role in the inhibition of bacterial adhesion in vivo. Another biomolecule that is considered to be a virulence factor and to play a key role in bacterial motility is the biosurfactant rhamnolipid, produced by the opportunistic bacterium P. aeruginosa ${ }^{24,25}$. While the presence of CPAC inhibits $P$. aeruginosa swarming motility, addition of rhamnolipid has been found to partially restore motility ${ }^{16}$. Thus, interaction between CPAC and rhamnolipid is one possible mechanism via which CPAC inhibits bacterial motility, and is worthy of further investigation. 
The purpose of this study is two-fold: (i) to evaluate potential limitations to the bioavailability of a cranberry derivative, namely, CPAC, by characterizing its extent of binding to human serum proteins, and (ii) to investigate the potential interfering role of CPAC against selected bacterial virulence factors (LPS and rhamnolipids) by measuring their extent of binding to CPAC. A quartz crystal microbalance with dissipation monitoring (QCM-D) is used to measure adsorption of three different human serum proteins (HSA, fibrinogen, and AAG) to a layer of CPAC formed on the surface of the sensor. The same approach is used to quantify binding of CPAC to LPS from two different uropathogens ( $E$. coli $\mathrm{O} 111: \mathrm{B} 4$ and $P$. aeruginosa 10 ) or rhamnolipids from $P$. aeruginosa. After demonstrating that all of these biomolecules bind to the CPAC coated surface, the Langmuir adsorption model is used to calculate relative binding affinities of the molecules for CPAC and other adsorption and thermodynamic parameters.

\section{MATERIALS AND METHODS}

\section{Solutions}

Phosphate buffered saline (PBS) solution was prepared by dissolving a PBS tablet (Sigma-Aldrich) in deionized (DI) water and was filtered using a $0.22 \mu \mathrm{m}$ membrane prior to use.

Dry cranberry proanthocyanidin (CPAC) powder from Vaccinium macrocarpon was isolated as described previously ${ }^{26}$ (obtained from A.B. Howell, Rutgers, Chatsworth, USA) and was dissolved in DI water at a concentration of $1 \mathrm{mg} / \mathrm{mL}$ to form a stock solution. This stock solution was filtered through a $0.22 \mu \mathrm{m}$ membrane, and stored at $4^{\circ} \mathrm{C}$ protected from light. The stock solution was diluted to a concentration of $100 \mu \mathrm{g} / \mathrm{mL}$ in PBS as needed. 
LPS from Escherichia coli O111:B4 (ELPS) and Pseudomonas aeruginosa 10 (PLPS), as well as HSA, fibrinogen, and AAG from human serum, were purchased from Sigma-Aldrich and dissolved in PBS to obtain stock solutions having a concentration of $1 \mathrm{mg} / \mathrm{mL}$. The molecular weights for the ELPS, PLPS, HSA, fibrinogen, and AAG were $10 \mathrm{kDa}, 10 \mathrm{kDa}, 66 \mathrm{kDa}, 340 \mathrm{kDa}$, and $40.8 \mathrm{kDa}$, respectively, as provided by the supplier. The stock solutions of LPS and proteins were stored at $4^{\circ} \mathrm{C}$ and kept for no longer than 1 week. R95 rhamnolipids (AGAE Technologies, LLC, Corvallis, USA) were stored as a stock solution of $61.2 \mu \mathrm{g} / \mathrm{mL}$ at $4^{\circ} \mathrm{C}$ until needed. The rhamnolipids are a mixture of mono- and di-rhamnolipid in a ratio of $\sim 1: 5$, and an average molecular weight of $626 \mathrm{~g} / \mathrm{mol}$. Stock solutions were diluted in PBS to the desired target concentration immediately prior to each experiment.

\section{Quartz Crystal Microbalance with Dissipation Monitoring (QCM-D)}

The QCM is a mass sensor with nanogram sensitivity that allows real-time quantitative analysis of biomolecule adsorption and interactions ${ }^{27}$. The study of biological interactions is an area of high interest in QCM applications and has been thoroughly reviewed elsewhere ${ }^{28,29}$. Particularly, the use of the QCM for quantification of protein interactions ${ }^{30-33}$, for investigation of the role of LPS and other cell surface appendages in bacterial adhesion ${ }^{34,35}$, and for measurement of adsorption of CPAC onto materials used in the manufacture of implantable medical devices (PVC and PTFE) ${ }^{9}$ have been demonstrated.

The QCM is based on quartz piezoelectric properties; an AC voltage applied across two gold electrodes on the quartz crystal induces an oscillating expansion and contraction of the crystal lattice 
at its resonant frequency or odd overtones thereof ${ }^{28}$. In the case of the QCM, a minute change in the mass on the quartz crystal surface is sufficient to affect the crystal oscillation and thus cause a detectable resonant frequency shift. In 1959, Sauerbrey reported what has now become known as the Sauerbrey equation describing the linear relationship between changes in mass on the crystal surface and shifts in the resonant frequency of a quartz crystal ${ }^{28,36}$ :

$$
\Delta m=-\frac{C}{n} \Delta f
$$

where $\Delta m$ is the change in mass at the crystal surface in $\mathrm{ngcm}^{-2}, \Delta f$ is the observed frequency shift in $\mathrm{Hz}, C$ is the mass sensitivity constant $\left(C=17.7 \mathrm{ngcm}^{-2} \mathrm{~Hz}^{-1}\right.$ for a $5 \mathrm{MHz}$ crystal), and $n$ is the odd overtone number, where $n=3$ was used for all calculations herein. In quartz crystal microbalance with dissipation monitoring (QCM-D), the energy dissipation is measured concurrently with frequency shifts. The dissipation of a crystal is deduced from the oscillation decay time and is mathematically defined as ${ }^{28}$ :

$$
D=\frac{E_{\text {dissipated }}}{2 \pi E_{\text {stored }}}
$$

where $D$ is the dimensionless dissipation factor, and $E$ is the energy being either dissipated or stored during one oscillation of the crystal system. Traditionally, the Sauerbrey equation is limited to rigid thin films growing at the crystal surface, precluding the study of liquid based biosystems. However, in this case, because the energy dissipation is sufficiently small in all experiments, that is, the $\Delta D / \Delta f$ values are less than $4 \times 10^{-7} \mathrm{~Hz}^{-1}$, the Sauerbrey equation can be used to evaluate the mass, and thus the surface concentration, of the biomolecules adsorbed to the CPAC coated crystal surface ${ }^{37}$. 
In this study, all QCM-D experiments were performed in an E4 QCM-D unit from Q-Sense (QSense AB, Göteborg, Sweden) using purchased AT-cut quartz crystals coated with titanium (QSX310) with a fundamental resonance frequency of $5 \mathrm{MHz}$. Titanium coated crystals were chosen as a model surface due to consistent and significant CPAC adsorption to the surface. Prior to each experiment, the crystals were cleaned by soaking in a $2 \%$ Hellmanex solution for at least $30 \mathrm{~min}$. They were then sonicated for $10 \mathrm{~min}$ in the $2 \%$ Hellmanex solution before being thoroughly rinsed with DI water and dried with high purity nitrogen gas. Finally, the crystals were exposed to ultraviolet light for $20 \mathrm{~min}$ immediately before the experiment.

A peristaltic pump (RegloDigital, Ismatec) was used to maintain the flow at negative pressure through the QCM-D modules at $150 \mu \mathrm{L} / \mathrm{min}$ for all experiments. The temperature was maintained at $22{ }^{\circ} \mathrm{C}$ throughout the experiments using the built-in temperature controller of the QCM-D. Frequency and dissipation baselines in DI water and in PBS were allowed to stabilize before each experiment.

After the establishment of a stable baseline in PBS, a CPAC solution $(100 \mu \mathrm{g} / \mathrm{mL})$ was flowed across the crystal surface to deposit a layer of CPAC on the crystal. This was followed by a 10 min rinse with PBS to remove any CPAC that was loosely bound to the crystal surface. The desired LPS, rhamnolipid, or protein solution was flowed across the CPAC-coated crystal until the deposition had reached equilibrium. Loosely bound molecules were again rinsed away with a PBS rinse of at least 10 min.

Following each experiment, the QCM-D setup was cleaned by injecting a $2 \%$ Hellmanex solution followed by DI water through the apparatus for at least $10 \mathrm{~min}$ each. The water was purged from the modules and tubing using air and nitrogen gas. All QCM-D experiments were performed in triplicate. 


\section{Determination of Biomolecule Binding Constants}

The equilibrium surface concentration of a biomolecule bound to the CPAC coated surface can be calculated from the QCM-D mass using the known molecular weight of the molecule. In the simplest case, the equilibrium surface concentration of an adsorbed biomolecule can be related to the biomolecule concentration in solution using the Langmuir adsorption isotherm ${ }^{33}$ :

$$
\Gamma=\frac{\Gamma_{\max } K C}{1+K C}
$$

where $C$ is the biomolecule solution concentration in $\mathrm{mol} / \mathrm{L}, \Gamma$ is the surface concentration of biomolecules in $\mathrm{mol} / \mathrm{cm}^{2}, \Gamma_{\max }$ is the maximum surface concentration of a monolayer of biomolecules in $\mathrm{mol} / \mathrm{cm}^{2}$, and $K$ is the binding affinity constant for the biomolecule-CPAC interaction in $\mathrm{L} / \mathrm{mol}$. The isotherm above can be linearized in the following manner ${ }^{38}$ :

$$
\frac{C}{\Gamma}=\frac{1}{\Gamma_{\max } K}+\frac{C}{\Gamma_{\max }}
$$

By plotting $C / \Gamma$ versus $C$ and fitting a straight line to the data, the maximum surface concentration and the thermodynamic binding affinity constant can be determined from the slope and intercept. Furthermore, the binding affinity constant, $K$, can be used to calculate the apparent Gibbs free energy of adsorption as follows ${ }^{38}$ :

$$
K=\frac{1}{c_{\text {solvent }}} \exp \left(\frac{-\Delta G_{a d s}}{R T}\right)
$$

where $K$ is the binding affinity constant for the biomolecule-CPAC interaction in $\mathrm{L} / \mathrm{mol}, c_{\text {solvent }}$ is the molar concentration of the solvent (herein, $c_{\text {solvent }}=c_{\text {water }}=55.5 \mathrm{molm}^{-3}$ ), $R$ is the gas constant in $\mathrm{Jmol}^{-1} \mathrm{~K}^{-}$ ${ }^{1}, T$ is the temperature in $\mathrm{K}$, and $\Delta G_{a d s}$ is the Gibbs free energy of adsorption in $\mathrm{Jmol}^{-1}$. While providing valuable information on biomolecule binding to the CPAC, the Langmuir isotherm must be used with 
caution as it depends on several assumptions: reversible adsorption, no lateral interactions on the surface, equal energy of all surface adsorption sites, and monolayer adsorption. Although several of these assumptions have been questioned in the case of protein adsorption, in this case, the Langmuir model is used simply for purposes of comparison between the different biomolecules. To limit inaccuracies in the Langmuir adsorption isotherm model due to protein spreading and enable comparison of the binding constants of the various biomolecules, direct QCM-D adsorption measurements for each protein concentration were used rather than sequential QCM-D measurements 33 .

\section{ATR-FTIR}

The adsorption of proteins to the QCM-D crystal surface was confirmed using a manual Bruker Hyperion attenuated total reflection Fourier transform infrared (ATR-FTIR) spectrometer. The protein spectra were collected over 120 scans at a spectral resolution of $4 \mathrm{~cm}^{-1}$ with an uncoated $\mathrm{Ti}$ QCM-D crystal used as a baseline. The spectra were collected from $5500 \mathrm{~cm}^{-1}$ to $800 \mathrm{~cm}^{-1}$; however, only the characteristic region for protein from $1800 \mathrm{~cm}^{-1}$ to $1400 \mathrm{~cm}^{-1}$ was further analyzed.

Samples for ATR-FTIR analysis were prepared using the same QCM-D procedure as that described in the previous section with some modifications. After protein adsorption had reached equilibrium and the crystals had been rinsed with PBS, the flow of PBS was stopped and the QCM-D modules were opened to remove the protein coated crystals. The QCM-D crystals were allowed to dry via evaporation for at least 3 hours. Crystals were further dried in a vacuum desiccator immediately before ATR-FTIR measurements in order to minimize the interference of water with the protein spectra. 
Three crystals each were coated with CPAC, CPAC and fibrinogen, and CPAC and HSA with measurements taken at three different locations on each crystal. Thus, each spectrum is an average of the 9 ATR-FTIR measurements.

\section{RESULTS AND DISCUSSION}

\section{Biomolecule Binding to a CPAC Coated Surface Monitored by QCM-D}

CPAC binds to the QCM-D crystal surface causing a negative frequency shift as mass adsorbs, as predicted by the Sauerbrey equation. Upon rinsing the CPAC coated surface with buffer, no desorption is observed indicating a stable CPAC surface coating. The frequency shift of a representative QCM-D experiment involving the adsorption of CPAC, a rinse in buffer, the adsorption of a protein to the CPAC layer, and a final rinse can be seen in Figure 1.

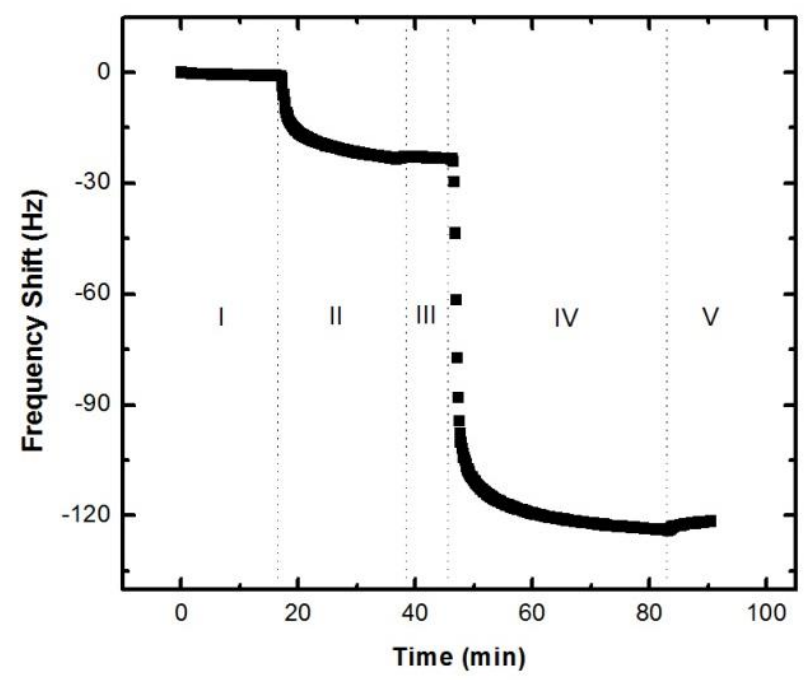

Figure 1.An example of the adsorption of CPAC directly on the crystal surface and the subsequent adsorption of fibrinogen to the CPAC coated surface. Five phases can be seen in the plot: (I) Baseline in PBS buffer, (II) Adsorption of the CPAC layer, (III) Rinse of the CPAC layer with PBS buffer, (IV) Adsorption of fibrinogen from a $1.5 \mu \mathrm{M}$ solution causing a negative frequency shift, (V) Rinse of the fibrinogen layer using PBS buffer. 
As exemplified in Figure 2, all of the biomolecules bind to the CPAC coated surface, causing a negative frequency shift proportional to the adsorbed mass. However, the molecules exhibit vastly different time-dependent adsorption behaviours, examples of which can be seen in Figure 2.
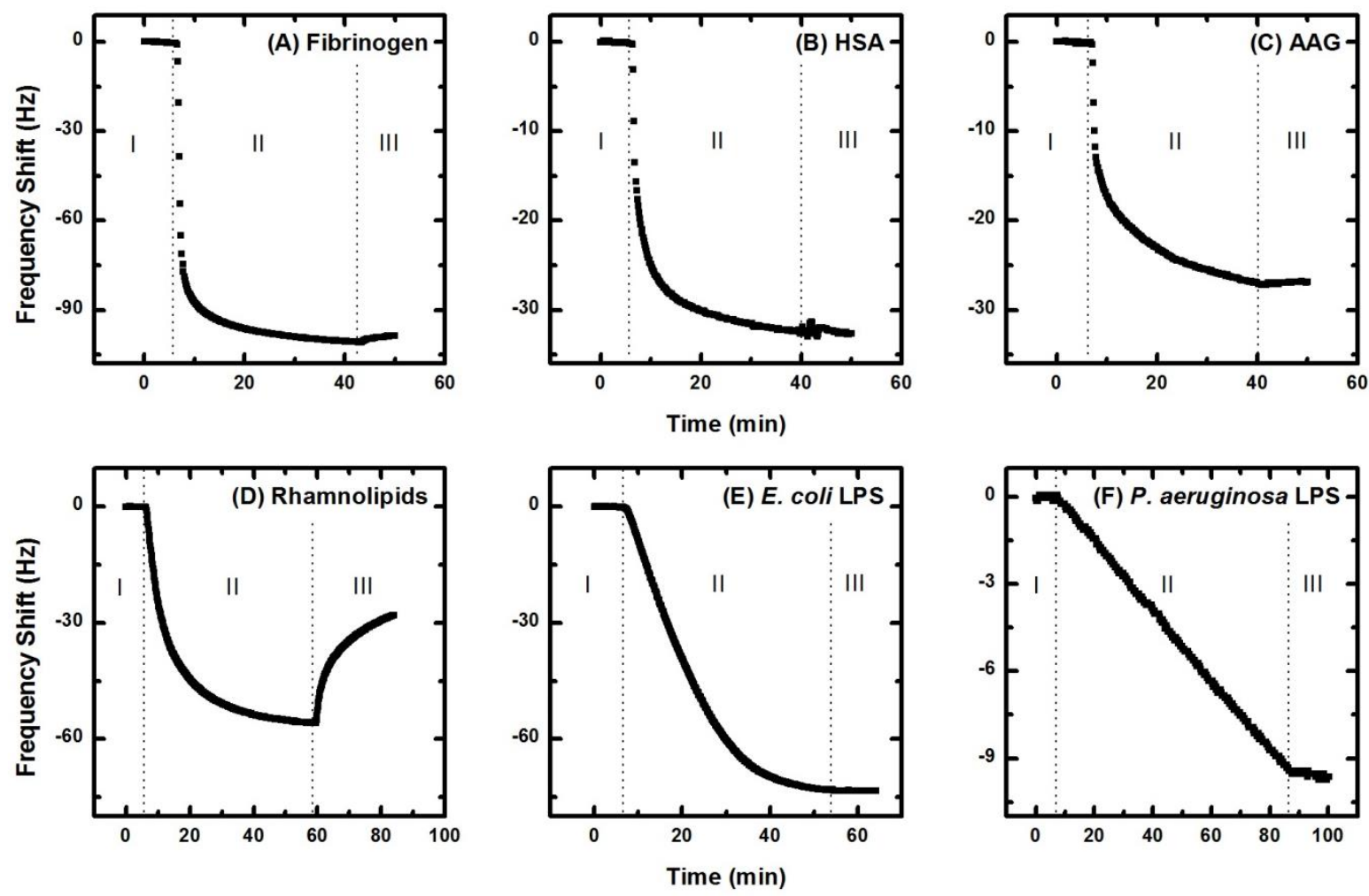

Figure 2.Representative examples of the time-dependent adsorption behaviours of the different biomolecules to the CPAC coated surface. The frequency shift in each example is referenced to the PBS rinse after CPAC adsorption; thus, only three phases are shown in the plots: (I) Rinse of the CPAC layer with PBS buffer, (II) Adsorption of the biomolecule from solution causing a negative frequency shift, and (III) Rinse of the adsorbed biomolecule layer with PBS buffer. The adsorption from $1.5 \mu \mathrm{M}$ solutions of (A) fibrinogen, (B) HSA, and (C) AAG and from $10 \mu \mathrm{g} / \mathrm{mL}$ solutions of (D) rhamnolipids, (E) ELPS, and (F) PLPS to the CPAC layer are shown.

For the selected proteins (Figure 2A-C), the initial rate of adsorption to CPAC is high and the rate of adsorption gradually decreases until an equilibrium surface concentration is reached, at which point the measured frequency shift remains constant with time. The presence of protein on the CPAC coated 
surface at equilibrium was confirmed using FTIR, which exhibited a dramatic increase in the characteristic protein peak in the amide I region due to the protein binding (see Supporting Information). Upon rinsing with buffer once equilibrium adsorption is reached, the frequency shifts for HSA and AAG remain relatively constant at their equilibrium values, indicating that the molecules are tightly bound to the $\mathrm{CPAC}$ and exhibit little desorption (Figure $2 \mathrm{~B}-\mathrm{C}$ ). In contrast, for the rhamnolipids, the frequency increases from its equilibrium value during the final PBS rinse, indicating that some molecules are desorbing (Figure 2D). Fibrinogen exhibits two distinct desorption regimes based on initial fibrinogen concentration in solution. At low concentration $(1.5 \mu \mathrm{M})$, fibrinogen behaves similarly to the other proteins and only minimal desorption occurs upon rinsing with buffer (Figure $2 \mathrm{~A})$, but at high concentration $(7.4 \mu \mathrm{M})$, there is significant desorption during the rinse step (see Supporting Information). It is suspected that this occurs because interactions or aggregation of fibrinogen molecules in solution at high concentration cause the fibrinogen to adsorb to the CPAC in a different orientation with a weaker binding interaction.

While the LPS molecules isolated from E. coli and P. aeruginosa both bind to the CPAC (Figure 2EF), they exhibit vastly different adsorption behaviour. ELPS binds more rapidly to CPAC than PLPS, displaying behaviour similar to the HSA and AAG proteins (Figure 2E). It reaches an equilibrium surface concentration and exhibits little desorption upon rinsing with buffer. The PLPS exhibits completely different time dependent adsorption behaviour from the other molecules investigated. While PLPS binds to the CPAC, it binds at a very slow constant rate and has a much smaller frequency shift over the course of the experiment than the other molecules. Furthermore, it does not appear to reach an equilibrium surface concentration in the typical 2 hour timescale investigated for these 
experiments (Figure 2F). Similar behaviour is also observed during experiments where PLPS is allowed to adsorb to the CPAC overnight, with no equilibrium being reached despite the longer timescale (see Supporting Information). Upon rinsing with buffer, the frequency remains constant, indicating that there is little desorption of the adsorbed PLPS. Because the binding of the PLPS is not observed to reach an equilibrium surface concentration, it is believed that multilayer binding is occurring with the first layer of PLPS binding to the CPAC and further layers binding to previously adsorbed PLPS.

The adsorption behavior of all molecules to the CPAC coated surface is within the Sauerbrey regime ${ }^{37}$, allowing the direct calculation of adsorbed mass and thus surface concentration. The ratio of $\Delta D / \Delta f$ at equilibrium adsorption can be used as an indication of the viscoelasticity of the equilibrium layer; this ratio is calculated for all adsorbed molecules except for PLPS, which never reached equilibrium. The $\Delta D / \Delta f$ ratio for the three proteins can be seen in Figure 3 .
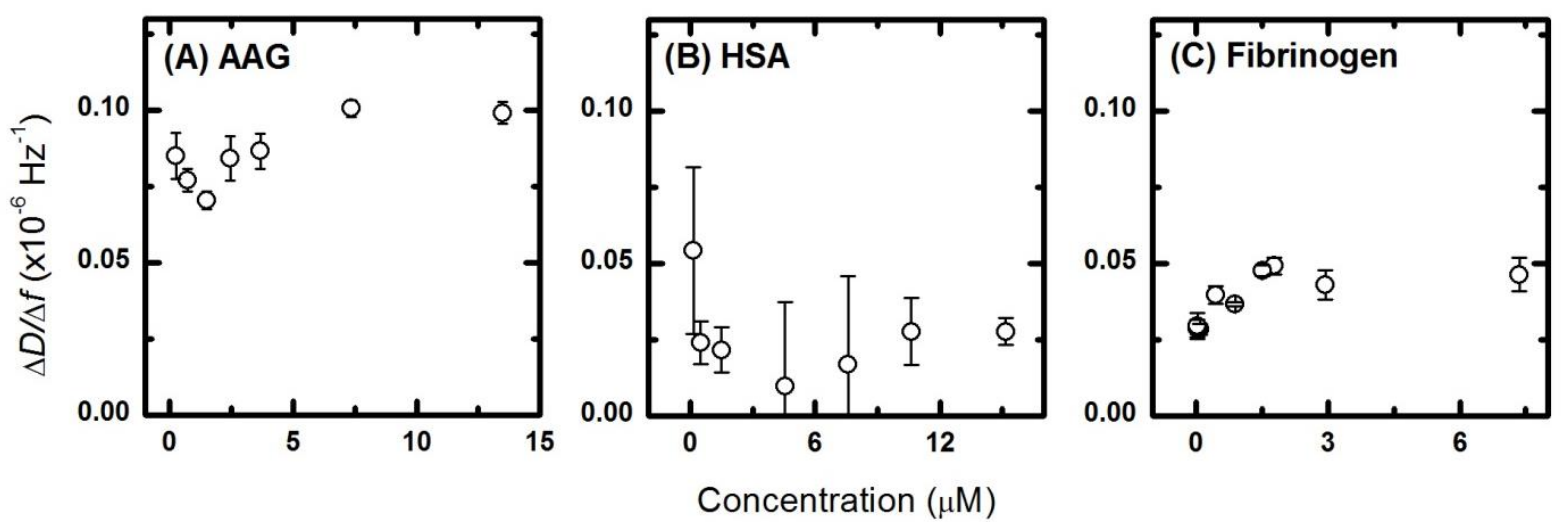

Figure 3.Equilibrium $\Delta D / \Delta f$ values calculated at different initial solution concentrations of (A) AAG, (B)HSA, and (C) fibrinogen. All three proteins show relatively low and relatively constant $\Delta D / \Delta f$ ratios. The data are averages of triplicate experiments.

For the proteins, the ratio of $\Delta D / \Delta f$ at equilibrium adsorption remains relatively low and relatively constant across all solution concentrations of protein investigated. This indicates that there is no major 
change in rigidity or trapped water in the adsorbed layer as the initial concentration of protein in solution is varied ${ }^{31}$.

The $\Delta D / \Delta f$ ratios of ELPS and rhamnolipid display distinctly different behaviour than that of the proteins (Figure 4), probably due to the amphiphilic nature of the molecules. LPS molecules consist of a hydrophobic lipid A section, a core section, and a long hydrophilic polysaccharide chain known as the $\mathrm{O}$-antigen ${ }^{23}$. The $\Delta D / \Delta f$ ratio of the ELPS equilibrium adsorption was higher than that of the proteins at all initial concentrations of ELPS in solution (Figure 4A). A larger $\Delta D / \Delta f$ ratio is typical of flexible, highly hydrated layers ${ }^{39}$. Thus, a higher $\Delta D / \Delta f$ ratio is expected for a layer of ELPS molecules with long hydrophilic O-antigen chains that can carry and trap water molecules as they adsorb to the CPAC. The rhamnolipid has two distinct $\Delta D / \Delta f$ regimes (Figure $4 \mathrm{~B}$ ). At low concentrations of rhamnolipid in solution, the $\Delta D / \Delta f$ ratio is quite low and comparable to the $\Delta D / \Delta f$ ratio of the proteins. However, at high rhamnolipid concentrations, despite rhamnolipid being a comparatively small molecule, the $\Delta D / \Delta f$ ratio shows a marked increase and approaches that of the ELPS.

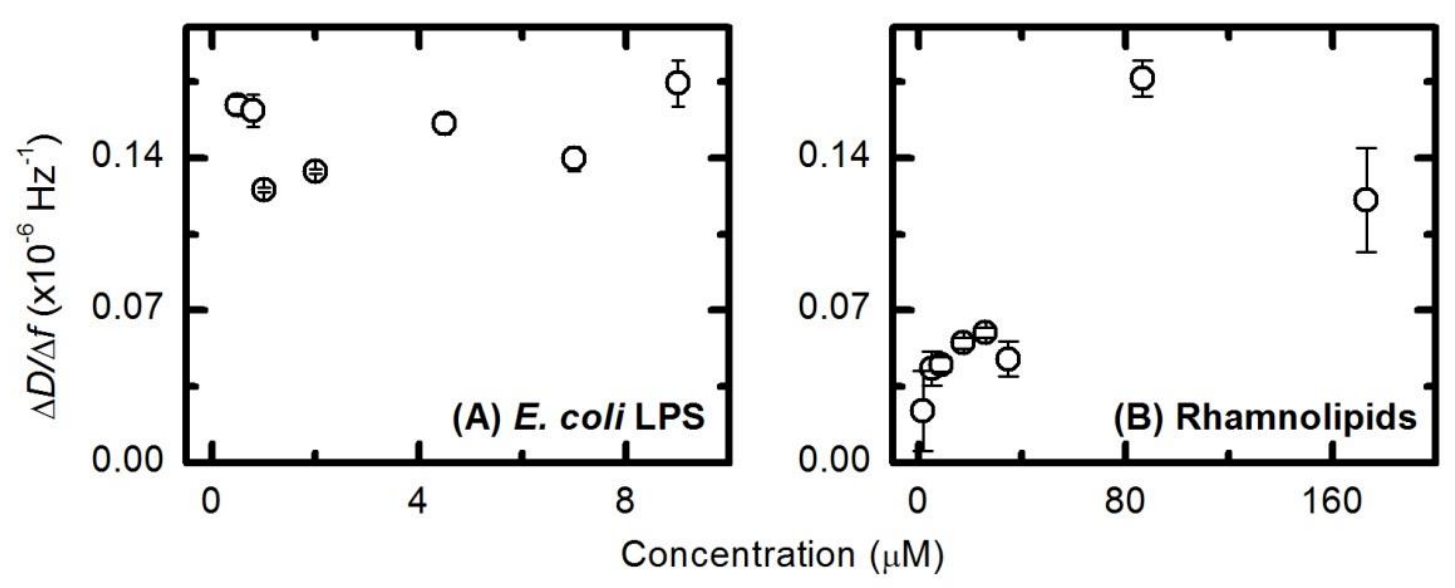

Figure 4.Equilibrium $\Delta D / \Delta f$ values calculated at different initial solution concentrations of (A) ELPS and (B) rhamnolipid. The ELPS shows a high but relatively constant $\Delta D / \Delta f$ ratio. The rhamnolipid 
shows a marked increase in $\Delta D / \Delta f$ ratio above the critical micelle concentration. The data are averages of triplicate experiments.

The adsorption behaviour of the ELPS and rhamnolipid must be analyzed taking into account the amphiphilic nature of these molecules. Both ELPS and rhamnolipids form micelles at concentrations above a critical micelle concentration (CMC) of $22 \mu \mathrm{g} / \mathrm{mL}^{40,41}$, or a molar concentration of about 2.2 $\mu \mathrm{M}$ for ELPS and $35 \mu \mathrm{M}$ for rhamnolipids. The CMC of the rhamnolipid occurs at a concentration between the adsorption regime with a low $\Delta D / \Delta f$ and the adsorption regime with a high $\Delta D / \Delta f$, suggesting a change in adsorbed species from small rhamnolipid molecules to large viscoelastic rhamnolipid micelles. However, for the ELPS, the $\Delta D / \Delta f$ ratio remains relatively constant for concentrations both above and below the CMC. This suggests that there is no change in the size or hydration of the binding species, that is, the micelles are not binding to the CPAC coated surface. This result supports the theory that CPAC binds primarily to the lipid A portion of LPS ${ }^{42}$; i.e., when the LPS is in micelle form it will not bind to the CPAC because the lipid A is embedded in the hydrophobic core of the micelle rendering it inaccessible. However, more rigorous experimentation is needed to completely rule out CPAC binding to LPS in micellar form, as other explanations exist for the micelles not binding such as mass transport effects or steric repulsion of the micelles by LPS molecules already bound to the CPAC coated surface.

\section{Langmuir Adsorption Isotherms}

The adsorption isotherms of the three proteins, the rhamnolipid, and the ELPS to the CPAC portray characteristic Langmuir adsorption behaviour and can be well described by the linearized Langmuir adsorption models, as exemplified by the fibrinogen isotherms (Figure 5). 

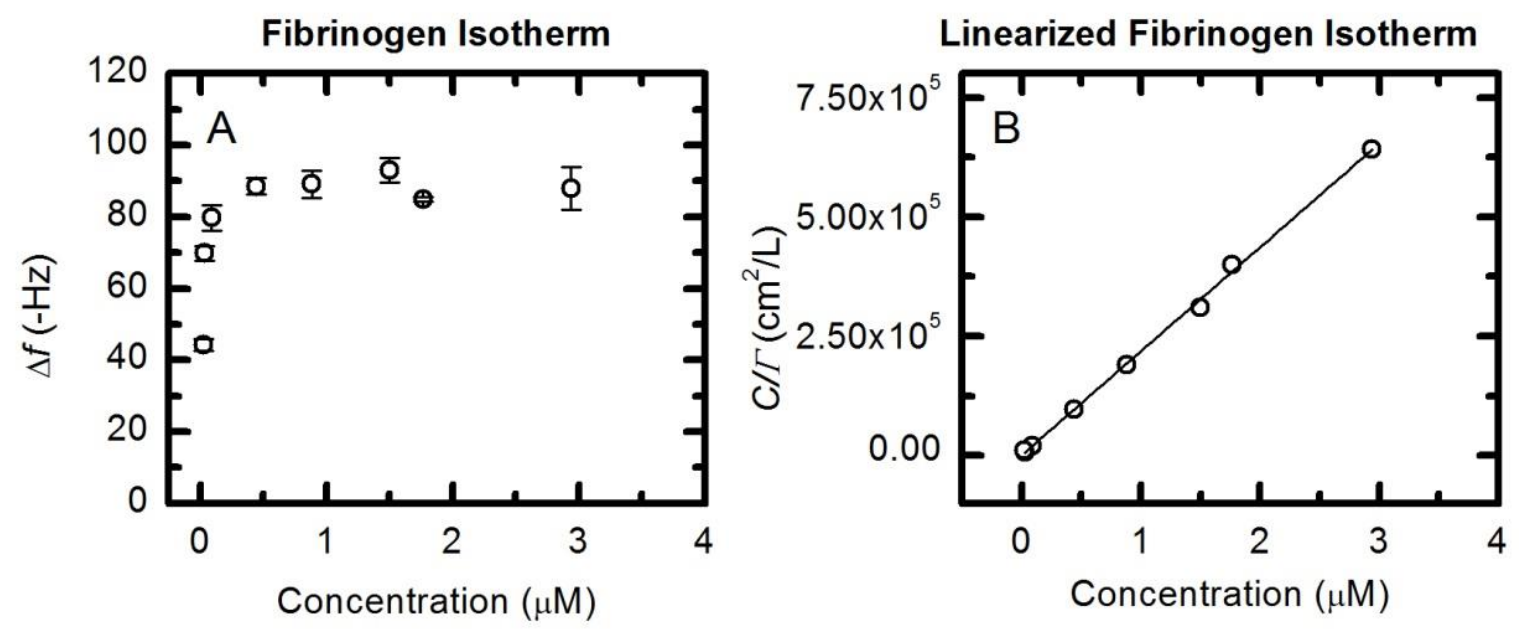

Figure 5. (A) The fibrinogen isotherm showing the frequency shift at equilibrium surface concentration of fibrinogen versus the initial fibrinogen concentration in solution, displays typical Langmuirian behaviour. (B) The linearized Langmuir isotherm for fibrinogen. The data are averages of triplicate experiments.

The representative isotherm (Figure 5A) shows that the amount of fibrinogen adsorbed to the CPAC at equilibrium increases as the concentration of fibrinogen in solution increases, until it reaches a maximum surface concentration plateau typical of Langmuir adsorption. At this point, increasing the fibrinogen solution concentration does not affect the amount of fibrinogen adsorbed to the CPAC at equilibrium. The linearized form of this isotherm (Figure 5B) allows the calculation of the maximum surface concentration, $\Gamma_{\max }$, the binding affinity constant, $K$, and $\Delta G_{\text {ads }}^{\circ}$ values, which are summarized for each biomolecule along with the fit of the linearized Langmuir model in Table 1.

Table 1.Summary of adsorption parameters calculated from linearized Langmuir isotherms

\begin{tabular}{|l|c|c|c|c|c|}
\hline Adsorbate & $M W(\mathrm{~g} / \mathrm{mol})$ & $\Gamma_{\max }\left(\mathrm{mol} / \mathrm{cm}^{2}\right)$ & $K\left(\mathrm{M}^{-1}\right)$ & $\Delta G^{\mathbf{o}}{ }_{a d s}(\mathrm{~kJ} / \mathrm{mol})$ & $R^{2}$ \\
\hline Fibrinogen & 340,000 & $4.6 \times 10^{-12}$ & $2.2 \times 10^{8}$ & -56.9 & 0.999 \\
\hline Albumin & 66,000 & $1.2 \times 10^{-11}$ & $2.4 \times 10^{6}$ & -45.9 & 0.995 \\
\hline AAG & 40,800 & $1.8 \times 10^{-11}$ & $1.5 \times 10^{6}$ & -44.7 & 0.994 \\
\hline
\end{tabular}




\begin{tabular}{|l|c|c|c|c|c|}
\hline ELPS & 10,000 & $1.4 \times 10^{-10}$ & $5.2 \times 10^{6}$ & -47.8 & 0.998 \\
\hline Rhamnolipids & $626^{a}$ & $1.6 \times 10^{-9}$ & $5.7 \times 10^{5}$ & -42.3 & 0.986 \\
\hline
\end{tabular}

${ }^{a}$ A weighted average molecular weight of mono and di-rhamnolipids is assumed ${ }^{43}$

Note: The data are averages of triplicate experiments. Adsorption parameters could not be evaluated for PLPS because no equilibrium adsorption was reached.

A comparison of the maximum surface concentrations of the five adsorbates shows that as the molecular weight of the biomolecule increases, the maximum surface concentration decreases, which is generally to be expected based on steric considerations. The apparent binding affinities for HSA, $\mathrm{AAG}$, and rhamnolipids to $\mathrm{CPAC}$ are in the same range as literature values reported for other protein binding interactions measured using the QCM-D ${ }^{33}$ and reported protein-drug binding affinities ${ }^{44,45}$. In contrast, the binding affinity of fibrinogen is considerably higher and is closer to the binding affinity for an antigen-antibody complex ${ }^{46}$. The apparent binding affinity of ELPS for CPAC is nearly 4 times larger than the reported value of about $1.43 \times 10^{6} \mathrm{M}^{-142}$; however, the LPS molecules are not necessarily comparable as they are from different $E$. coli serotypes ${ }^{23}$.

Finally, the negative $\Delta G_{\text {ads }}^{\mathrm{o}}$ values calculated from the isotherms thermodynamically confirm the spontaneous binding of all of the biomolecules to the CPAC as observed in the QCM experiments. While not expected to be identical to those in literature due to the different conditions used, the $\Delta G^{\circ}{ }_{a d s}$ values of $-45.9 \mathrm{kJmol}^{-1}$ and $-56.9 \mathrm{kJmol}^{-1}$ reported in this study for HSA and fibrinogen, respectively, are comparable to the range of -48 to $-59 \mathrm{kJmol}^{-1}$ reported for BSA and -40 to $-55 \mathrm{kJmol}$ ${ }^{1}$ reported for fibrinogen ${ }^{38}$. Moreover, the general trend of smaller proteins having a less negative $\Delta G^{\circ}{ }_{a d s}$ value, (i.e., a lower affinity for the surface), than larger proteins, is also observed in this study 38. 
The spontaneous binding between the serum proteins and CPAC, particularly the strong affinity with fibrinogen, could limit bioavailability of CPAC in the gut, urinary tract, bladder, or other sites of infection. This is a factor that should be taken into account when determining magnitude and frequency of dose for any future clinical trials involving the use of CPAC to prevent or treat bacterial infections. Furthermore, the spontaneous binding of rhamnolipids from $P$. aeruginosa to CPAC supports the hypothesis that CPAC could affect $P$. aeruginosa motility by interacting with the rhamnolipids ${ }^{16}$. However, the difference of an order of magnitude in the binding affinities of LPS from $E$. coli and rhamnolipids from $P$. aeruginosa, and the vastly different time-dependent binding behaviours of the different bacterial components to the CPAC, suggest that CPAC acts to prevent infections via different mechanisms and potentially with different effectiveness depending on the bacterium, another factor that should be taken into account before prescribing CPAC as a preventative measure against infections. Indeed, earlier studies conducted in the authors' laboratory show that CPAC exhibits different levels of bioactivity against the uropathogens E. coli CFT073, P. mirabilis, and $P$. aeruginosa ${ }^{16,18,19}$. Whereas CPAC can significantly impair both swimming and swarming motilities of E. coli CFT073 and P. mirabilis HI4320, in the case of $P$. aeruginosa, only the swarming motility is completely blocked ${ }^{16,18,19}$.

\section{CONCLUSION}

In this study, we confirm that QCM-D can be used to directly investigate the interactions between CPAC and biomolecules in real-time. Moreover, we demonstrate that CPAC binding to human serum proteins is a potential limitation to the bioavailability of CPAC and that CPAC plays an interfering 
role against the bacterial virulence factors LPS and rhamnolipids. Because individual binding affinities do not always reflect the outcome of competitive binding studies, additional research should investigate the time-dependent competitive binding of the biomolecules to build upon the results presented herein and provide additional clarity on the bioavailability and the mechanisms by which cranberry prevents bacterial infection. 


\section{SUPPORTING INFORMATION AVAILABLE}

ATR-FTIR spectra of QCM-D crystals coated with CPAC, CPAC and fibrinogen, and CPAC and HSA. An example of a QCM-D experiment involving fibrinogen at high concentration and PLPS over a long time period adsorbing to a CPAC coated surface. This material is available free of charge via the Internet at http://pubs.acs.org.

\section{ACKNOWLEDGEMENTS}

This research was financially supported by NSERC, the CRC Program, the Cranberry Institute, and the Wisconsin Cranberry Board. The authors would like to acknowledge Andreas Wargenau for technical assistance and Ché O’May for assistance with manuscript revision. 


\section{REFERENCES}

(1) Beerepoot, M. A. J.; ter Riet, G.; Nys, S.; van der Wal, W. M.; de Borgie, C. A. J. M.; de Reijke, T. M.; Prins, J. M.; Koeijers, J.; Verbon, A.; Stobberingh, E. Arch. Intern. Med. 2011, 171, 1270-1278.

(2) Howell, A. B. Mol. Nutr. Food Res. 2007, 51, 732-737.

(3) Serrano, J.; Puupponen-Pimiä, R.; Dauer, A.; Aura, A.-M.; Saura-Calixto, F. Mol. Nutr. Food Res.2009, 53, S310-S329.

(4) Hisano, M.; Bruschini, H.; Nicodemo, A. C.; Srougi, M. Clinics 2012, 67, 661-668.

(5) Côté, J.; Caillet, S.; Doyon, G.; Sylvain, J. F.; Lacroix, M. Crit. Rev. Food Sci. Nutr. 2010, 50, 666-679.

(6) Kratochwil, N. A.; Huber, W.; Müller, F.; Kansy, M.; Gerber, P. R. Biochem. Pharmacol.2002, $64,1355-1374$

(7) Hagerman, A. E.; Butler, L. G. J. Biol. Chem.1981, 256, 4494-4497.

(8) Artz, W. E.; Bishop, P. D.; Dunker, A. K.; Schanus, E. G.; Swanson, B. G. J. Agric. Food Chem.1987, 35, 417-421.

(9) Eydelnant, I. A.; Tufenkji, N. Langmuir 2008, 24, 10273-10281.

(10) Chan, M.; Hidalgo, G.; Asadishad, B.; Almeida, S.; Muja, N.; Mohammadi, M. S.; Nazhat, S. N.; Tufenkji, N. Colloids Surf. B 2013, 275-280.

(11) Ofek, I.; Goldhar, J.; Zafriri, D.; Lis, H.; Adar, R.; Sharon, N. N. Engl. J. Med. 1991, 324, 1599-1599.

(12) Habash, M.; Van der Mei, H.; Busscher, H.; Reid, G. Colloids Surf. B 2000, 19, 13-17.

(13) Ahuja, S.; Kaack, B.; Roberts, J. The Journal of Urology 1998, 159, 559-562.

(14) Foo, L. Y.; Lu, Y.; Howell, A. B.; Vorsa, N. J. Nat. Prod. 2000, 63, 1225-1228.

(15) Liu, Y.; Gallardo-Moreno, A. M.; Pinzon-Arango, P. A.; Reynolds, Y.; Rodriguez, G.; Camesano, T. A. Colloids Surf. B 2008, 65, 35-42.

(16) O'May, C.; Tufenkji, N. Appl. Environ. Microbiol. 2011, 77, 3061-3067.

(17) O'May, C.; Ciobanu, A.; Lam, H.; Tufenkji, N. Biofouling 2012, 28, 1063-1076.

(18) McCall, J.; Hidalgo, G.; Asadishad, B.; Tufenkji, N. Can. J. Microbiol. 2013, 59, 430-436.

(19) Hidalgo, G.; Chan, M.; Tufenkji, N. Appl. Environ. Microbiol. 2011, 77, 6852-6857.

(20) Hidalgo, G.; Ponton, A.; Fatisson, J.; O'May, C.; Asadishad, B.; Schinner, T.; Tufenkji, N. Appl. Environ. Microbiol. 2011, 77, 1532-1535.

(21) Lindhout, T.; Lau, P. C.; Brewer, D.; Lam, J. S. Microbiology 2009, 155, 3449-3460.

(22) Alexander, C.; Rietschel, E. T. J. Endotoxin Res. 2001, 7, 167-202.

(23) Erridge, C.; Bennett-Guerrero, E.; Poxton, I. R. Microbes Infect. 2002, 4, 837-851.

(24) Soberón-Chávez, G.; Lépine, F.; Déziel, E. Appl. Microbiol. Biotechnol. 2005, 68, 718-725.

(25) Zulianello, L.; Canard, C.; Köhler, T.; Caille, D.; Lacroix, J.-S.; Meda, P. Infect. Immun. 2006, 74, 3134-3147.

(26) Foo, L. Y.; Lu, Y.; Howell, A. B.; Vorsa, N. Phytochemistry 2000, 54, 173-181.

(27) Höök, F.; Kasemo, B.; Nylander, T.; Fant, C.; Sott, K.; Elwing, H. Anal. Chem. 2001, 73, $5796-5804$.

(28) Dixon, M. C. Journal of Biomolecular Techniques 2008, 19, 151-158.

(29) Janshoff, A.; Steinem, C. Sens. Update 2001, 9, 313-354.

(30) Ayela, C.; Roquet, F.; Valera, L.; Granier, C.; Nicu, L.; Pugniere, M. Biosens. Bioelectron.2007, $22,3113-3119$.

(31) Höök, F.; Rodahl, M.; Brzezinski, P.; Kasemo, B. Langmuir 1998, 14, 729-734. 
(32) Malmström, J.; Agheli, H.; Kingshott, P.; Sutherland, D. S. Langmuir 2007, 23, 9760-9768.

(33) Brewer, S. H.; Glomm, W. R.; Johnson, M. C.; Knag, M. K.; Franzen, S. Langmuir 2005, 21, 9303-9307.

(34) Strauss, J.; Kadilak, A.; Cronin, C.; Mello, C. M.; Camesano, T. A. Colloids Surf., B2010, 75, 156-164.

(35) Olsson, A. L.; van der Mei, H. C.; Busscher, H. J.; Sharma, P. K. Langmuir 2008, 25, $1627-$ 1632.

(36) Sauerbrey, G. Phys. A: Hadrons Nucl. 1959, 155, 206-222.

(37) Reviakine, I.; Johannsmann, D.; Richter, R. P. Anal. Chem. 2011, 83, 8838-8848.

(38) Cosman, N. P.; Roscoe, S. G. Langmuir 2004, 20, 1711-1720.

(39) Höök, F.; Rodahl, M.; Kasemo, B.; Brzezinski, P. Proc. Natl. Acad. Sci.1998, 95, 1227112276.

(40) Aurell, C. A.; Wistrom, A. O. Biochem. Biophys. Res. Commun. 1998, 253, 119-123.

(41) Samadi, N.; Abadian, N.; Ahmadkhaniha, R.; Amini, F.; Dalili, D.; Rastkari, N.; Safaripour, E.; Mohseni, F. A. Folia Microbiol. 2012, 57, 501-508.

(42) Delehanty, J. B.; Johnson, B. J.; Hickey, T. E.; Pons, T.; Ligler, F. S. J. Nat. Prod. 2007, 70, $1718-1724$.

(43) Abdel-Mawgoud, A. M.; Lépine, F.; Déziel, E. Appl. Microbiol. Biotechnol. 2010, 86, 13231336.

(44) Xuan, H.; Hage, D. S. Anal. Biochem. 2005, 346, 300-310.

(45) Frostell-Karlsson, Å.; Remaeus, A.; Roos, H.; Andersson, K.; Borg, P.; Hämäläinen, M.; Karlsson, R. J. Med. Chem. 2000, 43, 1986-1992.

(46) Piehler, J.; Brecht, A.; Giersch, T.; Hock, B.; Gauglitz, G. J. Immunol. Methods 1997, 201, 189-206. 
TABLE OF CONTENTS GRAPHIC

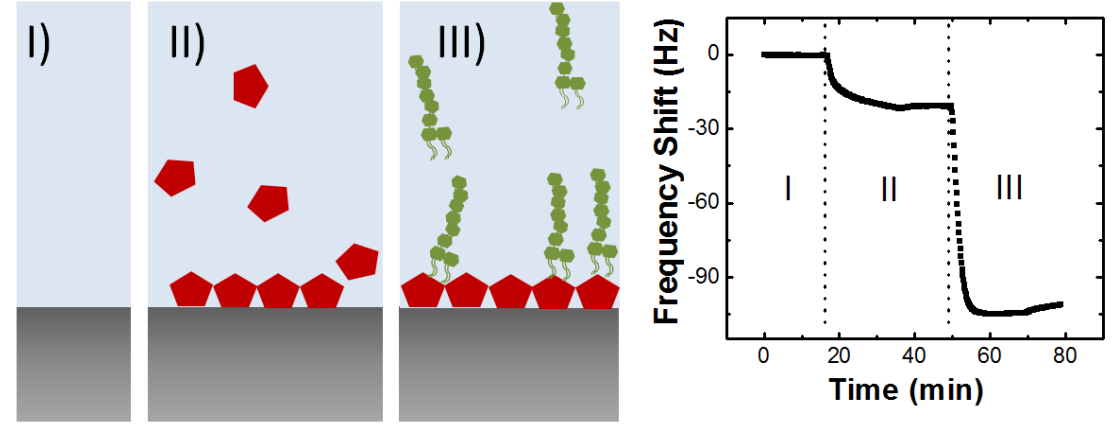

